\title{
Emotion regulation difficulties and interpersonal problems during the COVID-19 Pandemic: Predicting anxiety and depression
}

\author{
Ole André Solbakken ${ }^{1}$ \\ Omid V. Ebrahimi ${ }^{1,2}$ \\ Asle Hoffart ${ }^{2,1}$ \\ Jon T. Monsen ${ }^{1}$ \\ Sverre Urnes Johnson ${ }^{1,2}$ \\ ${ }^{1}$ Department of Psychology, University of Oslo \\ ${ }^{2}$ Research Institute, Modum Bad Psychiatric Hospital
}

\section{Citation:}

Solbakken, O. A., Ebrahimi, O. V., Hoffart, A., Monsen, J. T., \& Johnson, S. U. (2021, April 20). Emotion regulation difficulties and interpersonal problems during the COVID-19

Pandemic: Predicting anxiety and depression. Retrieved from psyarxiv.com/g5d8r https://doi.org/10.31234/osf.io/g5d8r

\section{Author Note}

A preprint of the paper has been posted online. Word count: 3902. The study was in its entirety funded internally by the University of Oslo, Norway. The authors declare no conflict of interest. Correspondence concerning this article should be addressed to Ole André Solbakken, Department of Psychology, University of Oslo, pb 1094 Blindern, N-0317 Oslo, Norway. E-mail: o.a.solbakken@psykologi.uio.no 


\begin{abstract}
Background: Central components of psychological functioning, such as difficulties in emotion regulation and interpersonal problems are likely to have been substantially impacted by COVID-19 and the amelioration measures of societal lock-down and social distancing. In turn, these factors are likely to predict mental health outcomes, such as symptoms of depression and anxiety throughout the pandemic and beyond.
\end{abstract}

Methods: To investigate this issue, we conducted an internet-based survey with 10,061 responders at the height of lock-down (T1). After social distancing measures had been eased (T2), 4,936 responders (49.1\%) completed the survey again.

Results: Emotion regulation difficulties, interpersonal problems, and symptoms of depression and anxiety decreased from T1 to T2, but changes were minor. After controlling for age and gender, emotion regulation- and interpersonal difficulties were associated with anxiety and depression throughout the study period, and changes in all domains occurred in concert. More extensive problems with emotion regulation at $\mathrm{T} 1$ predicted greater reductions in both symptom domains as amelioration measures were eased, while the converse was true for interpersonal problems. Furthermore, the impact of initial emotion regulation difficulties on subsequent changes in both anxiety-and depressive symptoms was dependent on the level of interpersonal difficulties, so that high interpersonal problem load at $\mathrm{T} 1$ reversed the effect of emotion regulation difficulties on symptom development.

Conclusions: The results suggest that emotion regulation- and interpersonal difficulties are highly central to mental health during the pandemic, and may be important targets for remediation to reduce mental health problems throughout the course of the pandemic and beyond. 


\section{General Scientific Summary}

This study suggests that emotion regulation difficulties and interpersonal problems have been significantly impacted by the COVID-19 pandemic and the social distancing measures used to impede transmission of the virus. In turn, these factors and changes in them have been systematically predictive of mental health outcomes during the pandemic. The results indicate that emotion regulation difficulties and interpersonal problems may be useful targets of intervention to alleviate the mental health strain of the pandemic.

Keywords: COVID-19, mental health, emotion, emotion regulation difficulties, interpersonal problems, depression, anxiety, longitudinal multilevel analyses. 


\section{Emotion regulation difficulties and interpersonal problems during the COVID-19}

\section{Pandemic: Predicting the Course of Mental Health Strain from Strict to Discontinued}

\section{Social Distancing Protocols}

The COVID-19 pandemic and social distancing protocols aimed to slow its transmission are having severe mental health consequences (Brooks et al., 2020; Ebrahimi, Hoffart \& Johnson, 2021; Hoffart, Johnson \& Ebrahimi, 2020; Holmes et al., 2020; Salari et al., 2020). The pandemic disease itself and widespread fear of it, social distancing measures and associated restrictions of personal freedoms, along with the relational-, psychosocial-, and financial strain brought about by these extraordinary circumstances have produced a surge of mental health problems across affected countries. Psychological distress has increased substantially in the general population, and the pandemic appears to constitute an unprecedented threat to mental health in high-, middle-, and low-income countries alike (Xiong et al., 2020). The pandemic, and the measures taken to combat it, will produce different mental health consequences depending on peoples' typical ways of reacting to stressful circumstances. Among processes potentially central to the exacerbation and persistence of such symptoms, personality-based processes such as difficulties in the experience and regulation of emotion and severity of interpersonal problems are particularly salient candidates. These psychological processes are strongly associated with psychological symptoms (e.g. Horowitz, 2004; Solbakken, Hansen, Havik, \& Monsen, 2011) and both are important targets in intervention systems for common mental health problems (e.g. Elkin, Parloff, Hadley, \& Autrey, 1985; Greenberg \& Pascual-Leone, 2006).

Although primarily defined as stable skills and styles, both difficulties tolerating and regulating one's emotions (Solbakken, Hansen, \& Monsen, 2011) and problems in one's way of relating to others (Horowitz, 2004) are likely to be somewhat impacted by the pandemic and amelioration measures of societal lock-down and social distancing. As amelioration 
measures in turn are relaxed, this impact may presumably diminish, gradually returning these factors to pre-crisis levels. Difficulties in emotion regulation and interpersonal problems are, in turn, likely to predict mental health outcomes, such as symptoms of depression and anxiety, throughout the pandemic and beyond, and the early levels of these factors will presumably predict later developments in symptom status. Similarly, reductions in emotion regulation- and interpersonal difficulties during the various phases of the outbreak will presumably coincide with concurrent reductions in psychological symptoms and reduced symptom levels at later stages. Thus, emotion regulation difficulties and interpersonal problems are likely to be systematically predictive of the course of mental health problems during the pandemic, and both may constitute useful targets of intervention for reducing the mental health strain of the pandemic and its amelioration procedures.

Previous studies have indicated long-term association between distancing strategies such as lockdown and quarantine and mental health problems (Brooks et al., 2020). Accordingly, it is likely that the exacerbations of mental health difficulties we have witnessed in recent months will persist to a disturbing degree, even after the pandemic itself is stamped out. As a consequence, there is an urgent need to identify psychological processes central to the exacerbation and persistence of symptoms during and after the pandemic, so that so that vulnerable individuals can be identified and knowledge gained on potentially useful targets of intervention.

Accordingly, the aim of the present study was to examine how these processes have developed from the height of the COVID-19 pandemic and as amelioration measures of societal lock-down and social distancing were subsequently relaxed. We then tested the importance of these processes as predictors of essential mental health domains, i.e. symptoms of depression and anxiety, during the passage of the first wave of the pandemic. 
In order to investigate this issue, we conducted an internet-based survey with 10,061 responders at Time 1 (T1 - a period of strict social distancing protocols) and 4,936 (49.1\%) at Time 2 (T2 - a period when the majority of distancing protocols were discontinued). We examined changes in emotion regulation difficulties, interpersonal problems from $\mathrm{T} 1$ to $\mathrm{T} 2$, and how $\mathrm{T} 1$ levels and changes from $\mathrm{T} 1$ and $\mathrm{T} 2$ in these variables were related to changes from $\mathrm{T} 1$ to $\mathrm{T} 2$ in depression and anxiety. We specifically investigated the following hypotheses:

Hypothesis $1(\mathrm{H} 1)$ : We postulate a significant decrease in emotion-regulation difficulties and interpersonal problems from $\mathrm{T} 1$ to $\mathrm{T} 2$.

Hypothesis 2 (H2): We postulate that the T1-level and changes from T1 to T2 in emotion-regulation difficulties and interpersonal problems will predict changes from $\mathrm{T} 1$ to $\mathrm{T} 2$ in anxiety and depression during the pandemic above and beyond other relevant factors such as age, gender, and education. In further exploratory analyses we will examine the interaction of emotion regulation difficulties and interpersonal problems in the prediction of symptomatic change.

\section{Method}

\section{Study Design and Participants}

The study was a longitudinal observational survey of the general adult Norwegian population during the COVID-19 pandemic (see Ebrahimi, Hoffart, \& Johnson, 2021; Hoffart et al., 2020 for further details). Eligible participants were individuals 18 years and older living in Norway and who provided informed consent. Participants experienced identical social distancing protocols implemented nationally. These protocols consisted of not leaving home unless necessary, home isolation if infected, quarantine after exposure to possible infection, closure of kindergartens, schools, universities, and other public spaces, restrictions on traveling, and prohibitions of social gatherings and arrangements. Strict distancing 
protocols were implemented in Norway on March 12th 2020. The first period of data collection for the study lasted seven days and was between March 31st 2020 and April 7th 2020 (T1). The strict protocols were upheld during the nearly three weeks prior to data collection, as well as during the data collection week. No new information was given by the government during this period regarding changes of distancing protocols, keeping expectation effects constant.

In total, 10,061 persons completed the first round of the survey. On June 8th 2020, the Norwegian government informed about upcoming removal of most of the social distancing protocols, and from June 15th, the protocols were largely discontinued. Discontinuation included re-opening of kindergartens, schools, universities, and workplaces, the opportunity for international travel was resumed, and minor cultural events were allowed. However, people were still keeping distance ( 1 meter) to others, wearing masks if necessary, and restricting gatherings to no more than 20 people.

A second wave of data were collected from the sample starting one week after the discontinuation of strict lockdown, i.e. from 22 th of June and lasting three weeks to July 13 th (T2). From the original sample of 10,061, a total of 4,936 (49.1\%) responded. The new social distancing protocols were held constant during the entire assessment period. At T1, the number of new infected cases per day was 196 (March 31st) and 221 (April 7th). At T2, the numbers were 6 (June 22th) and 3 (July 13th) new cases (Worldometers, 2020).

Ethical approval of the study was granted by The Regional Committee for Medical and Health Research Ethics in Norway and it was formally registered in The Norwegian Centre for Research Data (reference numbers: 125510 and 802810, respectively). The preregistered protocol for the study - submitted prior to data collection - is available from Clinicaltrials.gov (Identifier: NCT04442282). The study is part of The Norwegian COVID-19 Mental Health and Adherence Project (Ebrahimi, Hoffart, \& Johnson, 2021). 


\section{Procedures}

As described in detail elsewhere (e.g. Ebrahimi et al., 2021), the survey was distributed online with the aim of giving the complete adult population of Norway equal opportunity to participate. The survey was disseminated in six ways. 1) Through a Facebook Business algorithm, with imputed parameters covering a population of 3.6 million adults, proportionately targeting each geographical region compared to their relative size. The total number of individuals reached encompassed a random selection of 174,885 of these 3.6 million individuals.

As Facebook includes approximately $85 \%$ of the Norwegian adult population (i.e., 3.6 out 4.2 million), five other methods were used to maximize the probability of reaching the residual $15 \%$ of the adult population (i.e., 600,000 individuals); 2 ) through broadcasting on the national, public news channel of Norway, with approximately 1.1 million viewers at the time of broadcast. We also disseminated information about the survey through 3) national radio stations; 4) regional and local radio stations across the country; 5) national newspapers; and 6) regional and local newspapers across the country, as well as additional local and regional media and social media sources. These national, regional, and local announcements contained brief messages informing about the opportunity to participate in a University of Oslo-study about mental health during the pandemic in the adult population.

Of the 10,061 participants, almost $70 \%$ were obtained randomly among the pool of adults on Facebook (i.e., $85 \%$ of the population), while $30 \%$ were obtained by platforms 2 to 6 designed to reach the residual $15 \%$ of Norwegian adults. The dissemination procedure is described in greater detail elsewhere (Ebrahimi et al., 2021).

\section{Measures}


The survey included questions about demographic and social variables and about whether one have mainly stayed at home since the distancing measures were implemented on March 12th (see Table 1).

Emotion regulation difficulties was assessed by a subset of 6 items from the Difficulties in Emotion Regulation Scale (DERS; Gratz \& Roemer, 2004). Items were selected by expert consensus and based on the principle of minimizing topological/conceptual overlap between items. Items are rated on a scale from 1 ("almost never") to 5 ("almost always"). Higher scores indicate more difficulty in emotion regulation. Cronbach's alpha for the scale was .74 at $\mathrm{T} 1$ and .77 at $\mathrm{T} 2$.

Interpersonal problems was assessed by a subset of 17 items from the Inventory of Interpersonal Problems-64 (IIP-64; Horowitz, Alden, Wiggins, \& Pincus, 2000). Items were chosen by expert consensus through the selection of 2-3 items from each of the IIP- subscales deemed most central to internalizing problems. Items denote distressing interpersonal behaviors rated as "hard to do" (i.e., behavioral inhibitions) or "does too much" (i.e., behavioral excesses) on a 0 ("not at all") to 4 ("very much") scale. Higher scores indicate more extensive relational problems. Cronbach's alpha for the scale was .81 at T1 and .84 at $\mathrm{T} 2$.

Symptoms of depression was assessed by The Patient Health Questionnaire-9 (PHQ9; Kroenke, Spitzer, \& Williams, 2001). It consists of nine items covering the DSM-IV criteria for major depression scored on a four-point 0 ("not at all”) to 3 ("almost every day") scale. Cronbach's alpha for the scale was .88 at T1 and .91 at T2.

Symptoms of anxiety was assessed by The Generalized Anxiety Disorder-7 (GAD-7; Spitzer, Kroenke, Williams, \& Löwe, 2006). This scale consists of seven items covering the DSM-IV criteria for GAD scored on a four-point 0 ("not at all") to 3 ("almost every day") scale. Cronbach's alpha was .88 at T1 and .90 at T2. 


\section{Statistical Analyses}

The statistical analyses were done with SPSS 26.0 (IBM Corp, 2018). Repeated surveys like this usually, and as this case did, have high drop-out rates and accompanying missing data. Therefore, hierarchical linear mixed (multilevel) models were used to analyze the data. Multilevel models use maximum likelihood estimation, which is the state-of-the-art approach to handling missing data (Schafer \& Graham, 2002). If data are missing at random (MAR), which is likely in the present study, such models give more unbiased results than other analytic strategies (O'Connel et al., 2017). Given the large sample size in this study, a conservative level of $p<.01$ was used to determine significance. Effect sizes are presented as Cohen's $d$. Standards for evaluating the effect sizes were adopted from Cohen (1988): small, $d>=0.20-0.50 ;$ medium, $d>=0.50-0.80 ;$ and large, $d>0.8$.

In preparatory analyses for each of the predictor and dependent variables - emotion regulation difficulties (DERS), interpersonal problems (IIP), symptoms of depression (PHQ), symptoms of anxiety (GAD) - the combination of random effects and covariance structure of residuals that gave the best fit for the "empty" model (the model without fixed predictors except the intercept) was chosen. The Akaike Information Criterion (AIC) was used to compare the fit of different models. Models that gave a reduction in AIC greater than 2 were considered better (Burnham \& Anderson, 2004).

Testing of specific hypotheses were done as follows. First, H1 about decrease in emotion regulation difficulties, interpersonal problems, anxiety- and depression symptoms was tested through separate multilevel models with DERS, IIP, GAD, and PHQ as dependent variables with time $(\mathrm{T} 1$ period $=0, \mathrm{~T} 2$ period $=1)$ as a predictor. We then tested the various components of $\mathrm{H} 2$ through separate multilevel models with GAD and PHQ as dependent variables with stepwise addition of the relevant predictor variables. In the second step, the 
demographic variables of age and gender, along with their respective interactions with time were added (testing the effects of age and gender on the level and development of symptoms). In the third step, initial DERS- and IIP-levels were added, in addition to the interaction of these respective covariates and time (testing the effects of initial DERS and IIP on the level and development of symptoms). In the fourth step, we explored the interaction between initial DERS and IIP levels, along with their three-way interaction with time (testing interaction effects of those covariates on the level and development of symptoms). Finally, the T2 levels of DERS and IIP were added, together with their respective interactions with time (testing the relationship of changes in DERS and IIP on the level and development of symptoms).

\section{Results}

\section{Descriptive statistics}

Mean age of the 10,061 respondents at $\mathrm{T} 1$ was 36.0 years $(S D=13.5)$. The remaining 4,936 respondents at T2 had a mean age of 38.9 years $(S D=13.8)$. See Table 1 for central sample characteristics at T1 and T2. In total, 17\% of the sample had lost their job (laid off, dismissed) due to COVID-19 at T1, but only $3 \%$ had a pandemic-related job loss at T2. Descriptive statistics for predictor and outcome variables at T1 and T2 are displayed in Table 2.

\section{Preliminary multilevel analyses}

Preparatory multilevel analyses demonstrated that the combination of random effects and covariance structures of residuals that gave the best fit for the "empty" models varied according to outcome variable. Anxiety symptoms $(\triangle \mathrm{AIC}=-15.86)$ and interpersonal problems $(\triangle \mathrm{AIC}=-12.10)$ were more effectively analyzed using a heteroscedastic error covariance structure, while depression symptoms $(\triangle \mathrm{AIC}=1.84)$ and emotion regulation 
difficulties $(\triangle \mathrm{AIC}=1.05)$ were more effectively analyzed using the statistically simpler homoscedastic error covariance structure (see Tables 3 and 4 for details of analyses).

The development of emotion regulation difficulties, interpersonal problems, depression symptoms, and anxiety symptoms from $\mathrm{T} 1$ to $\mathrm{T} 2$.

The results of the multilevel models testing $\mathrm{H} 1$ showed significant time effects on emotion regulation difficulties, interpersonal problems, symptoms of anxiety, and symptoms of depression (see Table 3). As can be seen, emotion regulation difficulties, interpersonal problems, symptoms of depression, and symptoms of anxiety all demonstrated significant reductions across time. Figure 1 displays these reductions in terms of effect sizes. In line with Cohen (1988), the effects for all four problem domains could be classified as minor or less than small.

Predicting the level and course of depression- and anxiety symptoms.

Multilevel models testing $\mathrm{H} 2$ and its components can be found in Tables 4 and 5 . Addition of the demographic variables of age, gender and education, along with their interactions with time (Model 1) showed that males, older persons, and the more highly educated had lower depression scores at T1. Males and older persons reported smaller reductions in depression to $\mathrm{T} 2$. Likewise, males, older persons and the more highly educated had lower anxiety at $\mathrm{T} 1$, and males reported smaller reductions in anxiety to T2.

The addition of initial emotion regulation difficulty- and interpersonal problem levels and their interactions with time (Model 2), showed that greater problem load in both domains was associated with more extensive anxiety- and depressive symptoms at T1. More extensive emotion regulation difficulties at $\mathrm{T} 1$ predicted greater reductions in both symptom domains, while more extensive interpersonal problems did not.

The addition of three-way interactions between emotion regulation difficulties, interpersonal problems, and time (Model 3), indicated that in both symptom domains the 
effect of high levels of initial emotion regulation difficulties on symptom reduction was dependent on the level of interpersonal problems. Thus, more pervasive interpersonal problems reversed the effect of emotion regulation difficulties on symptom development.

The final step, adding T2-levels of emotion regulation difficulties and interpersonal problems (Model 4) and their respective interactions with time, demonstrated that for both symptom domains reductions in the predictor variables across time were strongly associated with reductions in symptoms.

\section{Discussion}

The aim of this study was to examine the relationships between emotion regulation difficulties and interpersonal problems and the development of anxiety-and depressive symptoms in the general adult population when COVID-19-related social distancing protocols were discontinued (T2) following a period of strict protocols (T1). First, the levels and changes of predictor- and outcome variables were examined. Then a series of predictive multilevel models were computed testing specific hypotheses about the importance of emotion regulation difficulties and interpersonal problems as predictors of depression and anxiety, above and beyond demographic factors, from the height of lock-down to the first substantial easing of social distancing measures.

As hypothesized (H1), the results supported that problem load in all of the examined domains were significantly reduced, but with effect sizes $(d=0.05$ to 0.20$)$ that were minor according to standard criteria (Cohen, 1988). This modest change is also reflected in the high observed stability in of scores from $\mathrm{T} 1$ to $\mathrm{T} 2$, with correlations across time ranging from $\mathrm{r}=$ .69 to $r=.80$. The fact that changes were meager despite ordinary routines and social life to some extent returning to normal in Norway from T1 to T2, raises concerns that mental health impacts of COVID-19 may remain pervasive for an extended period of time after the eventual end of the pandemic, as has also been found from previous pandemics (e.g., Brooks et al., 
2020). Thus, vaccination, mass immunity and subsequent return to normal daily life may not in and of themselves lead to the desired rapid improvement of mental health in the population.

In terms of changes from $\mathrm{T} 1$ to $\mathrm{T} 2$, interpersonal problems and emotion regulation difficulties were characterized by more stability than the symptom scales. This may reflect a state-trait division, in which interpersonal- and emotional functioning constitute processes that are more aligned with personality-oriented dimensions, while anxiety and depression by definition are primarily referring to more state-like phenomena. Regardless of this, as demonstrated by the respective covariance parameters, significant inter-individual variability in levels at and changes from T1 to T2 was present in all four domains. Thus, sufficient variability in scores and trajectories was present to make predictive analyses and the potential resulting gains in understanding of these interrelated processes fruitful.

As with $\mathrm{H} 1$, tests of $\mathrm{H} 2$ and its components were in line with our expectations. While there were significant contributions of both gender and age on the level and course of symptoms, so that male gender and higher age served as protective factors for mental health throughout the study period, there were substantial effects of emotion regulation difficulties and interpersonal problems above and beyond these demographic factors. As was expected, greater problem load in both predictor domains was associated with more anxiety- and depressive symptoms across time, adding to the substantial literature demonstrating the centrality of emotional- and relational functioning in mental health outcomes (Gross, 2008; Solbakken, Hansen, Havik, et al., 2011; Widiger \& Smith, 2008).

Similarly, in line with our expectations, improvements in both predictor domains were associated with improvements in symptoms. This finding is consistent with the proposal that ongoing difficulties in accepting, processing, and tolerating emotions on the one hand (Tomkins, 2008), and maladaptive relational strategies on the other, have contributed to 
maintaining symptoms of anxiety and depression during the pandemic, and may be causal agents in the remediation such symptomatology. Thus, focused interventions that target these domains may help remediate the mental health strain associated with COVID-19.

Contrary to our hypothesis, more extensive emotion regulation difficulties initially predicted greater reductions in both symptom domains, while the opposite was true for interpersonal problems. Thus, participants with more initial emotion regulation difficulties became more similar to average responders in symptom distress from $\mathrm{T} 1$ to $\mathrm{T} 2$, while those with more initial interpersonal problems became further removed from the average in the same period. We may speculate that responders with greater difficulties tolerating unpleasant emotions were more negatively affected by the onset of the pandemic and lockdown, and also experienced more relief when the emotional pressures associated with COVID-19 and lockdown somewhat dissipated with increased control over the contagion and easing of the social distancing protocols. Likewise, the impeding effect of interpersonal problems on symptom improvement is theoretically reasonable, as more entrenched, maladaptive interpersonal strategies presumably contributed to hindering the constructive use of available social contacts in the service of improving one's situation and mental state as social distancing measures were eased. These propositions are also consistent with the observed interaction between emotion regulation difficulties and interpersonal problems on symptom improvement, i.e. more severe difficulties tolerating unpleasant emotions were associated with less symptom improvement when combined with more severe interpersonal problems. In this case, the additional relief afforded by reduced emotional pressure through eased amelioration measures for responders with low tolerance for unpleasant emotions was partly offset by the presence of persistent maladaptive relational strategies. Thus, even though lockdown was largely suspended at $\mathrm{T} 2$, unpleasant emotions aroused by extensive relational 
difficulties combined with poor capacity for dealing adaptively with these emotions (Solbakken \& Monsen, in press) impeded symptom improvement.

Limitations of this study included the following. Self-selection may have resulted in participants having specific characteristics that affected the results. However, effort was taken so that the entire adult population had equal opportunity to participate, and the resulting sample appears to be relatively representative (Ebrahimi et al., 2021). Second, there was substantial drop-out and consequently much missing data at T2. Again, self-selection may have influenced results. This issue was addressed through analyzing the data with an estimation method that gives the least possible bias in results if data are missing at random (O'Connel et al., 2017). Third, the full potential of multilevel longitudinal modelling could not be harnessed in our analyses, as assessments were available at only one or two occasions. However, the analytic approach chosen here is still the most suitable given the nature of the data (Schafer \& Graham, 2002).

To summarize, the discontinuation of the more intrusive social distancing measures and the re-opening of society seemed to have only minor or small effects on symptoms of anxiety and depression, emotion regulation difficulties, and interpersonal problems. Emotion regulation difficulties at the time of strict social distancing predicted greater reduction in symptoms from this period to the time of discontinued distancing. The opposite was true for interpersonal problems. Also, the predictive effect of early emotion regulation difficulties was reversed when combined with more severe interpersonal problems. Reductions in emotion regulation difficulties and interpersonal problems during the study period were related to concurrent reductions in symptom distress. Although the causal status of the examined covariates of anxiety and depressive symptoms is uncertain, our results tentatively suggest that tolerance of unpleasant emotions and maladaptive relational strategies are potential targets of intervention worth pursuing. It is likely that increased availability of 
psychotherapeutic treatments targeting these processes would be beneficial in alleviating anxiety and depression during the pandemic. Future studies should investigate whether increased symptom load extends beyond the COVID-19 pandemic, and whether the covariates identified in the present study are factors in post-pandemic persistence of mental health problems. 


\section{References}

Brooks, S.K., Webster, R.K., Smith, L.E., Woodland, L., Wessely, S., Greenberg, N., \& Rubin, G.J. (2020). The psychological impact of quarantine and how to reduce it: rapid review of the evidence. The Lancet, 395(10227), 912-920. http://dx.doi.org/10.1016/S0140-6736(20)30460-8

Burnham, K. P., \& Anderson, D. R. (2004). Multimodel inference: Understanding AIC and BIC in model selection. Sociological Methods \& Research, 33, 261-304. http://dx.doi.org/10.1177/0049124104268644

Cohen, J. (1988). Statistical power analysis for the behavioral sciences. Lawrence Erlbaum Associates.

DiGiovanni, C., Conley, J., Chiu, D., \& Zaborski, J. (2004). Factors influencing compliance with quarantine in Toronto during the 2003 SARS outbreak. Biosecurity and bioterrorism: biodefense strategy, practice, and science, 2, 265-272. http://dx.doi.org/10.1089/bsp.2004.2.265

Ebrahimi, O. V., Hoffart, A., \& Johnson, S. U. (2021, in press). Physical distancing and mental health during the COVID-19 pandemic: Factors associated with psychological symptoms and adherence to pandemic mitigation strategies. Clinical Psychological Science, in press. Preprint available at: https://doi.org/10.31234/osf.io/kjzsp

Elkin, I., Parloff, M. B., Hadley, S. W., \& Autry, J. H. (1985). NIMH treatment of Depression Collaborative Research Program: Background and research plan. Archives of general psychiatry, 42(3), 305-316.

Fitzmaurice, G. M., Laird, N. M., \& Ware, J. H. (2004). Applied longitudinal analysis. New York: Wiley.

Gratz, K. L., and Roemer, L. (2004). Multidimensional assessment of emotion regulation and dysregulation: development, factor structure, and initial validation of the difficulties 
in emotion regulation scale. J. Psychopathol. Behav. Assess. 26, 41-54. https://doi.org/10.1023/B:JOBA.0000007455.08539.94

Greenberg, L. S., \& Pascual-Leone, A. (2006). Emotion in psychotherapy: A practice friendly research review. Journal of clinical psychology, 62(5), 611-630.

Gross, J. J. (2008). Emotion regulation. Handbook of emotions, 3(3), 497-513.

Hoffart, A., Johnson, S. U., \& Ebrahimi, O. (2020). Loneliness and social distancing during the COVID-19 pandemic: Risk factors and associations with psychopathology. PsyArXiv. https://doi.org/10.31234/osf.io/j9e4q

Holmes, E. A., O'Connor, R. C., Perry, V. H., Tracey, I., Wessely, S., Arseneault, L.,....., (2020). Multidisciplinary research priorities for the COVID-19 pandemic: a call for action for mental health science. The Lancet Psychiatry, 7, 547-560. http://dx.doi.org/10.1016/S2215-0366(20)30168-1

Horowitz, L. M. (2004). Interpersonal foundations of psychopathology. American Psychological Association.

Horowitz, L. M., Alden, L. E., Wiggins, J. S., and Pincus, A. L. (2000). Inventory of Interpersonal Problems (IIP-32/IIP-64). London: Psychological Corporation.

IBM Corp. Released 2018. IBM SPSS statistics for windows. Version 26.0. Armonk, NY. IBM Corp. https://www-01.ibm.com/support/docview.wss?uid=swg21476197

Johnson SU, Ulvenes PG, Øktedalen T, Hoffart A. (2019). Psychometric properties of the GAD-7 in a heterogeneous psychiatric sample. Frontiers in Psychology, 10:1713. http://dx.doi.org/10.1016/S2215-0366(20)30168-1

Kroenke, K:, Spitzer, R. L., \& Williams, J. B. (2001). The PHQ-9: validity of a brief depression severity measure. Journal of General Internal Medicine, 16, 606-613. http://dx.doi.org/10.1016/S2215-0366(20)30168-1 
O’Connell, N. S., Dai, L., Jiang, Y., Speiser, J. L., Ward, R., Wei, W., ....., Gebregzabher, M. (2017). Methods for analysis of pre-post data in clinical research: A comparison of five common methods. Journal of Biometrics and Biostatistics, 8, 334. http://dx.doi.org/10.4172/2155-6180.1000334

Salari, N., Hosseinian-Far, A., Jalali, R., Vaisi-Raygani, A., Rasoulpoor, S., Mohammadi, M., ... Khaledi-Paveh, B. (2020). Prevalence of stress, anxiety, depression among the general population during the COVID-19 pandemic: a systematic review and meta-analysis.

Globalization and health, 16(1), 57-57. doi:10.1186/s12992-020-00589-w.

Schafer, J. L., Graham, J. W. (2002). Missing data: Our view of the state of the art. Psychological Methods, 7, 147-177. http://dx.doi.org/10.1037/1082-989X.7.2.147

Solbakken, O. A., Hansen, R. S., \& Monsen, J. T. (2011). Affect integration and reflective function: Clarification of central conceptual issues. Psychotherapy Research, 21(4), 482-496. https://doi.org/10.1080/10503307.2011.583696

Solbakken, O. A., Hansen, R. S., Havik, O. E., \& Monsen, J. T. (2011). Assessment of affect integration: Validation of the affect consciousness construct. Journal of personality assessment, 93(3), 257-265. https://doi.org/10.1080/00223891.2011.558874

Solbakken, O. A. \& Monsen, J. T. (In press). Validation of the Affect Integration Inventory Short Form (AII-SF-42). International Journal of Psychology and Psychological Therapy.

Spitzer, R. L., Kroenke, K., Williams, J. B., \& Löwe, B. (2006). A brief measure for assessing generalized anxiety disorder: the GAD-7. Archives of internal medicine, 166(10), 1092-1097.

Tomkins, S. S. (2008). Affect imagery consciousness: The complete edition: Two Volumes. Springer publishing company.

Widiger, T. A., \& Smith, G. T. (2008). Personality and psychopathology. In O. P. John, R. 
W. Robins, \& L. A. Pervin (Eds.), Handbook of personality: Theory and research (p. 743-769). The Guilford Press.

Worldometers. COVID-19 PANDEMIC. Coronavirus update (Live). (2020). Available online at: http://www.worldometers.info/coronavirus/Google Scholar

Xiong, J., Lipsitz, O., Nasri, F., Lui, L. M., Gill, H., Phan, L., ... \& McIntyre, R. S. (2020). Impact of COVID-19 pandemic on mental health in the general population: A systematic review. Journal of affective disorders. 
Table 1. Demographic and Social Variables for the Original Sample at T1 and for the Completer Sample at $T 2$

$\mathrm{T} 1$

No.

$10,061 \quad 100$

$4,936 \quad 100$

All participants

\section{Gender}

Female

Male

Transgender

Intersex

Age groups

$18-30$

$31-44$

$45-64$

$65+$

\section{Educational level}

High school or lower

$4,417 \quad 44$

$2,712 \quad 35$

University degree

$5,644 \quad 56$

$3,224 \quad 65$

Partnership status

Unmarried and not in a civil union

5,310

53

2,337

47

Married or in a civil union

4,75

47

$2,599 \quad 53$

\section{Employment status}

Currently unemployed

$1,928 \quad 19$

$1,156 \quad 23$

Currently employed

$8,140 \quad 81 \quad 3,780 \quad 77$


Refugee status

Refugee

Not refugee

\section{Children}

Having children

Not having children

\section{Psychological diagnosis}

Having a diagnosis

Not having a diagnosis

\section{Living status}

Living alone

Living with others

\section{Home confinement}

Mostly stayed home

Not mostly stayed home

\section{Employment and COVID-19}

Lost job due to COVID-19 1,367 17

8,694

$4,253 \quad 42$

$8,340 \quad 83$

Not lost job due to COVID-19
$2,420 \quad 49$

$5,808 \quad 58$

$2,516 \quad 51$

$1,721 \quad 17$

$890 \quad 18$

4,046

82

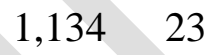

$4,046 \quad 77$

$\begin{array}{llll}7,952 & 79 & 3,892 & 79\end{array}$

$2,109 \quad 21 \quad 1,044 \quad 21$
2034

$4,733 \quad 96$

$\overline{\text { Note. }} \mathrm{T} 1 \mathrm{=}$ a period of one week (March 31st - April 7th 2020) starting nearly three weeks after the implementation of strict social distancing protocols in Norway (March 12th 2020). $\mathrm{T} 2=$ a period of three weeks (June 22th - July 13th 2020) starting one week after the strict social distancing protocols had been discontinued (June 15th 2020). 
Table 2. Descriptive Statistics for the Predictor and Outcome Variables across Time

\begin{tabular}{lccccccc}
\hline & T1 & \multicolumn{3}{c}{ T2 } & & & \\
Variable (range) & M & SD & M & SD & r & $\alpha$ \\
Emotion regulation difficulties (6-30) & 12.64 & 4.30 & 12.22 & 4.27 & .73 & .75 \\
Interpersonal problems (0-60) & 22.92 & 9.21 & 22.53 & 9.65 & .80 & .82 \\
Depression symptoms (0-27) & 7.32 & 5.69 & 6.63 & 5.66 & .74 & .91 \\
Anxiety symptoms (0-21) & 5.55 & 4.62 & 4.66 & 4.37 & .69 & .90 \\
\hline
\end{tabular}

Note. $\mathrm{T} 1=$ a period of one week $($ March 31st - April 7 th 2020) starting nearly three weeks after the implementation of strict social distancing protocols in Norway (March 12th 2020). $\mathrm{T} 2=$ a period of three weeks (June 22th - July 13th 2020) starting one week after the strict social distancing protocols had been discontinued (June 15th 2020).

$r=$ Pearson's $r . d=$ Cohen's $d . \alpha=$ Cronbach's $\alpha$. 
Figure 1. Effect Sizes of Changes from 11 to T2 in Anxiety Symptoms, Depression Symptoms, Emotion Regulation Difficulties, and Interpersonal Problems.

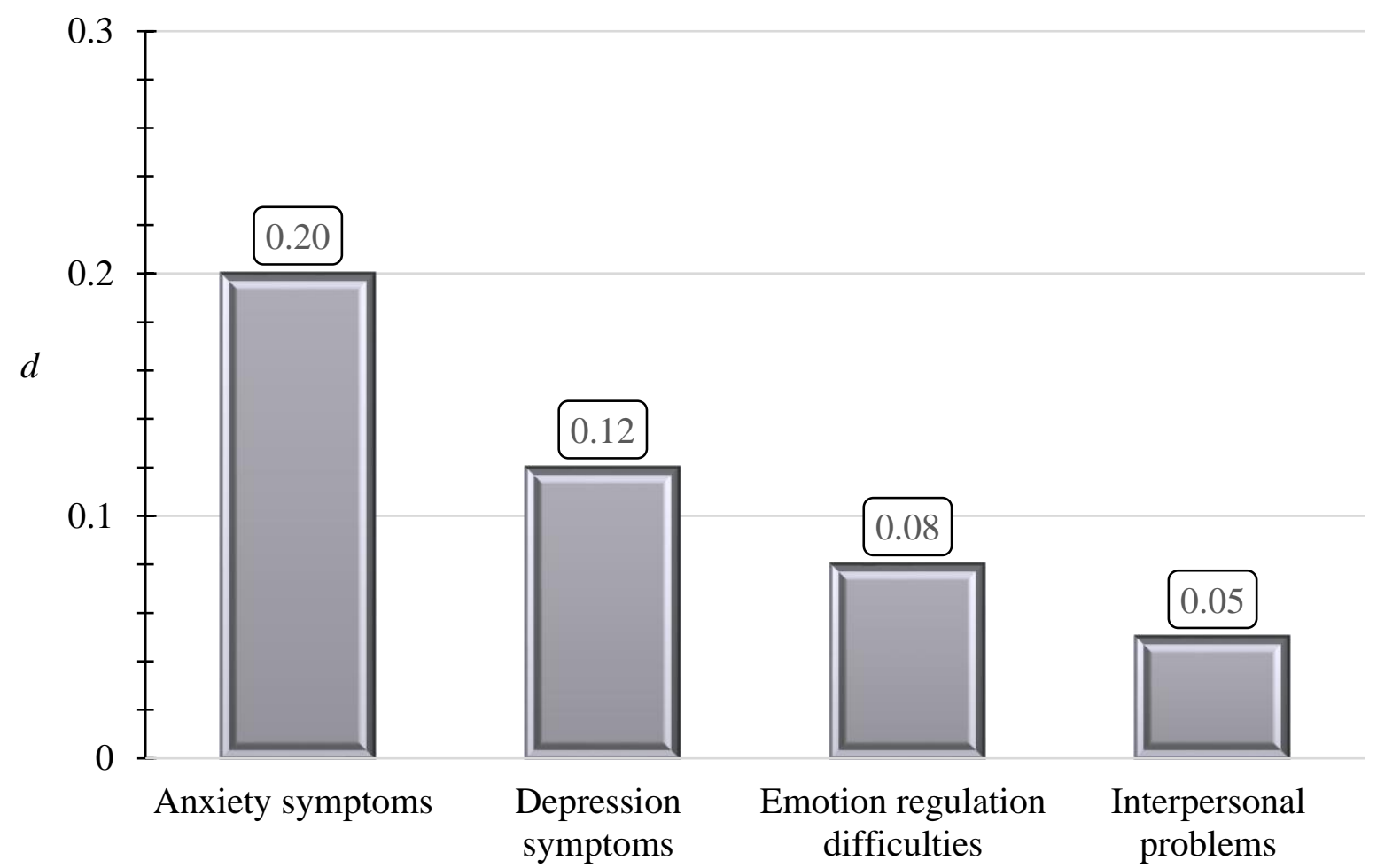

Note. $\mathrm{T} 1=$ a period of one week $($ March 31 st - April 7th 2020) starting nearly three weeks after the implementation of strict social distancing protocols in Norway (March 12th 2020). T2 $=$ a period of three weeks (June 22th - July 13th 2020) starting one week after the strict social distancing protocols had been discontinued (June 15th 2020). $d=$ Cohen's $d$. 
Table 3. Fixed Effects Estimates (Top) and Variance-Covariance Estimates (Bottom) for Multilevel Models of Difficulties in Emotion Regulation, Interpersonal Problems, Depressive Symptoms, and Anxiety Symptoms from $T 1$ to T2.

\begin{tabular}{|c|c|c|c|c|}
\hline & DERS & IIP & PHQ-9 & GAD-7 \\
\hline & Estimate & Estimate & Estimate & Estimate \\
\hline \multicolumn{5}{|l|}{ Fixed effects } \\
\hline Intercept & $12.64 *(0.04)$ & $22.92 *(0.09)$ & $7.61 *(0.06)$ & $5.73 *(0.05)$ \\
\hline \multirow[t]{2}{*}{ Time } & $-0.27 *(0.04)$ & $-0.44 *(0.08)$ & $-0.77 *(0.06)$ & $-0.95 *(0.05)$ \\
\hline & Estimate & Estimate & Estimate & Estimate \\
\hline \multicolumn{5}{|l|}{ Random effects } \\
\hline Variance intercept & $13.36 *(0.25)$ & $69.59 *(1.22)$ & $23.83 *(0.44)$ & $13.85 *(0.29)$ \\
\hline Residual Tot/T1 & $5.03 *(0.10)$ & $15.18 *(0.76)$ & $8.51 *(0.17)$ & $7.52 *(0.23)$ \\
\hline Residual T2 & & $20.40 *$ & - & $5.23 *(0.22)$ \\
\hline AIC & 82542.82 & 104477.2 & 90835.14 & 84793.99 \\
\hline
\end{tabular}

Note. Standard errors are given in parenthesis. Estimations were done by the method of maximum likelihood (ML). $* \mathrm{p}<.01$. Tot $=$ total residual in models with homoscedastic error covariance structures. T1 $=$ a period of one week (March 31st - April 7th 2020) starting nearly three weeks after the implementation of strict social distancing protocols in Norway (March 12th 2020). T2 = a period of three weeks (June 22th - July 13th 2020) starting one week after the strict social distancing protocols had been discontinued (June 15th 2020). DERS $=$ difficulties in emotion regulation. $\mathrm{IIP}=$ overall interpersonal problems. $\mathrm{PHQ}-9=$ symptoms of depression. GAD-7 = symptoms of anxiety. AIC $=$ Akaike's Information Criterion. 
Table 4. Fixed Effects Estimates (Top) and Variance-Covariance Estimates (Bottom) for

Predicitve Multilevel Models of Depressive Symptoms (PHQ-9) from T1 to T2.

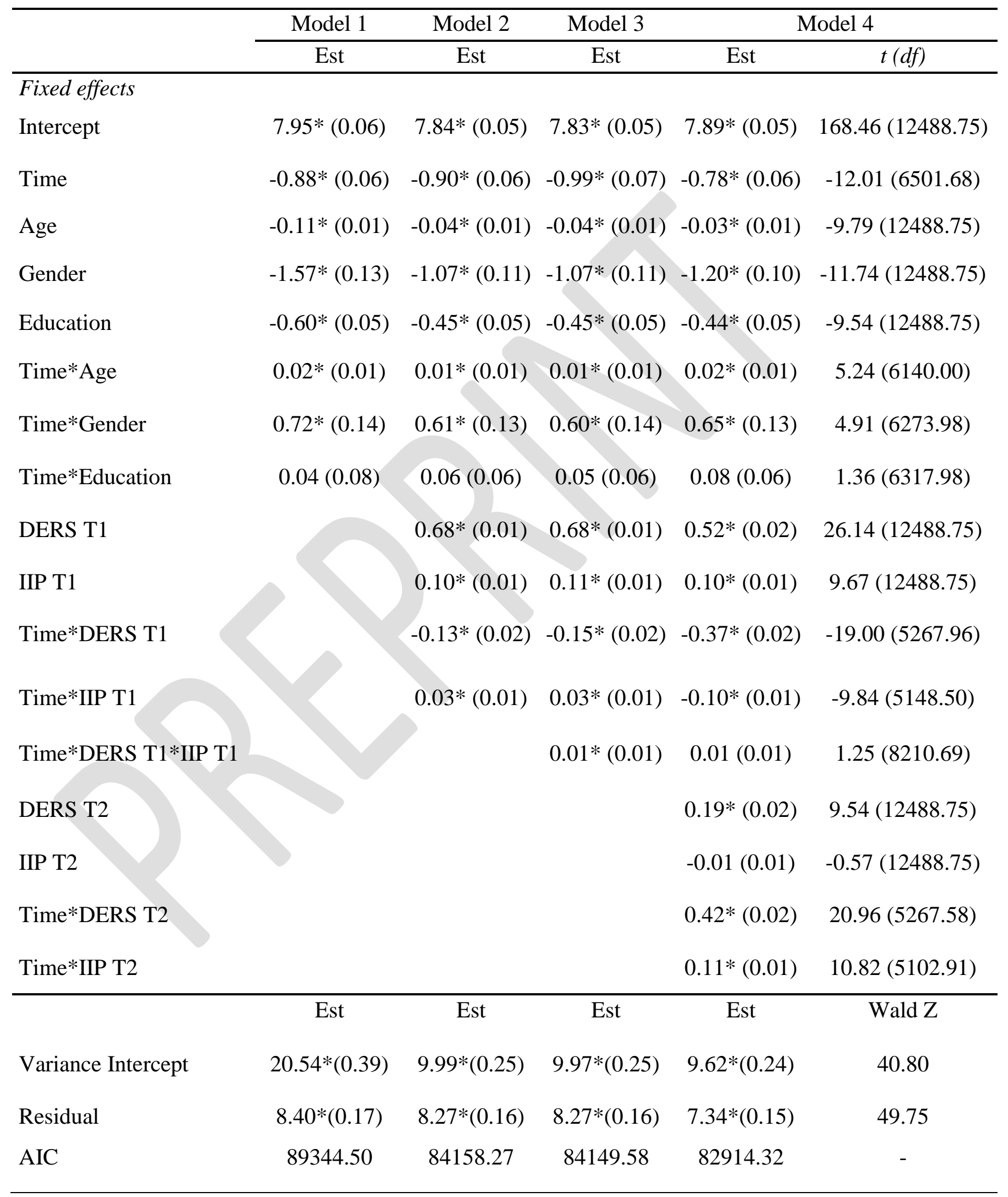


Note. Standard errors and degrees of freedom are given in parenthesis. Estimations were done by the method of maximum likelihood (ML) and with a homoscedastic error covariance structure. ${ }^{*} \mathrm{p}<.01 . \mathrm{T} 1=$ a period of one week (March 31st - April 7th 2020) starting nearly three weeks after the implementation of strict social distancing protocols in Norway (March 12th 2020). T2 = a period of three weeks (June 22th - July 13th 2020) starting one week after the strict social distancing protocols had been discontinued (June 15th 2020). DERS = difficulties in emotion regulation. IIP $=$ overall interpersonal problems. AIC $=$ Akaike's Information Criterion. Degrees of freedom $(d f)$, t-values, and Wald $\mathrm{Z}$ are given only for the final model. 
Table 5. Fixed Effects Estimates (Top) and Variance-Covariance Estimates (Bottom) for

Predictive Multilevel Models of Anxiety Symptoms (GAD-7) from T1 to T2.

\begin{tabular}{|c|c|c|c|c|c|}
\hline & \multirow{2}{*}{$\begin{array}{c}\text { Model } 1 \\
\text { Est } \\
\end{array}$} & \multirow{2}{*}{$\frac{\text { Model } 2}{\text { Est }}$} & \multirow{2}{*}{$\begin{array}{c}\text { Model } 3 \\
\text { Est }\end{array}$} & \multicolumn{2}{|c|}{ Model 4} \\
\hline & & & & Est & $t(d f)$ \\
\hline \multicolumn{6}{|l|}{ Fixed effects } \\
\hline Intercept & $6.08 *(0.05)$ & $5.97 *(0.04)$ & $5.97 *(0.04)$ & $5.99 *(0.04)$ & $148.33(10035.00)$ \\
\hline Time & $-1.01 *(0.05)$ & $-1.03 *(0.05)$ & $-1.10 *(0.06)$ & $-0.94 *(0.05)$ & $-17.43(5972.01)$ \\
\hline Age & $-0.06 *(0.01)$ & $-0.01 *(0.01)$ & $-0.01 *(0.01)$ & $-0.01 *(0.01)$ & $-4.90(10035.00)$ \\
\hline Gender & $-1.67 *(0.11)$ & $-1.15 *(0.09)$ & $-1.15^{*}(0.09)$ & $-1.15 *(0.09)$ & $-13.06(10035.00)$ \\
\hline Education & $-0.60 *(0.05)$ & $-0.17 *(0.04)$ & $-0.17 *(0.04)$ & $-0.16^{*}(0.04)$ & $-4.11(10035.00)$ \\
\hline Time*Age & $-0.01(0.01)$ & $-0.01(0.01)$ & $-0.01(0.01)$ & $0.01(0.01)$ & $0.70(5969.33)$ \\
\hline Time*Gender & $0.78 *(0.12)$ & $0.66 *(0.12)$ & $0.66^{*}(0.12)$ & $0.67 *(0.11)$ & $6.08(5982.96)$ \\
\hline Time*Education & 0.05( & $0.01(0.05)$ & $0.01(0.05)$ & $0.04(0.05)$ & $0.71(5984.17)$ \\
\hline DERS T1 & & $0.58 *(0.01)$ & $0.58 *(0.01)$ & $0.46^{*}(0.02)$ & $26.62(10035.00)$ \\
\hline IIP T1 & & $0.05 *(0.01)$ & $0.05 *(0.01)$ & $0.05 *(0.01)$ & $5.14(10035.00)$ \\
\hline Time*DERS T1 & & $-0.14 *(0.01)$ & $-0.15^{*}(0.01)$ & $-0.34 *(0.02)$ & $-19.86(5509.21)$ \\
\hline Time*IIP & & $0.03 *(0.01)$ & $0.03 *(0.01)$ & $-0.07 *(0.01)$ & $-7.48(5399.94)$ \\
\hline Time*DERS T $1 *$ IIP T1 & & & $0.01 *(0.01)$ & $0.01(0.01)$ & $1.34(4921.00)$ \\
\hline DERS & & & & $0.16^{*}(0.02)$ & $8.96(10035.00)$ \\
\hline IIP T2 & & & & $-0.01(0.01)$ & $-0.65(10035.00)$ \\
\hline Time*DERS T2 & & & & $0.34 *(0.02)$ & $19.87(5508.79)$ \\
\hline \multirow[t]{2}{*}{ Time*IIP T2 } & & & & $0.07 *(0.01)$ & $8.55(5355.75)$ \\
\hline & Est & Est & Est & Est & Wald Z \\
\hline Variance Intercept & $12.36 *(0.27)$ & $6.50 *(0.18)$ & $6.49 *(0.18)$ & $5.87 *(0.16)$ & 36.39 \\
\hline Residual T1 & $7.28 *(0.23)$ & $6.24 *(0.18)$ & $6.25 *(0.18)$ & $6.76^{*}(0.17)$ & 40.16 \\
\hline Residual T2 & $5.35 *(0.22)$ & $6.14 *(0.19)$ & $6.12 *(0.19)$ & $4.33 *(0.16)$ & 27.48 \\
\hline AIC & 83621.91 & 78987.70 & 78979.62 & 77825.80 & - \\
\hline
\end{tabular}


Note. Standard errors and degrees of freedom are given in parenthesis. Estimations were done by the method of maximum likelihood (ML) and a heteroscedastic error covariance structure. $* \mathrm{p}<.01 . \mathrm{T} 1=$ a period of one week (March 31st - April 7th 2020) starting nearly three weeks after the implementation of strict social distancing protocols in Norway (March 12th 2020). $\mathrm{T} 2=\mathrm{a}$ period of three weeks (June 22th - July 13th 2020) starting one week after the strict social distancing protocols had been discontinued (June 15th 2020). DERS $=$ difficulties in emotion regulation. IIP $=$ overall interpersonal problems. AIC $=$ Akaike's Information Criterion. Degrees of freedom $(d f)$, t-values, and Wald $\mathrm{Z}$ are given only for the final model. 
Figure captions.

Figure 1. Effect Sizes of Changes from T1 to T2 in Anxiety Symptoms, Depression Symptoms, Emotion Regulation Difficulties, and Interpersonal Problems. 